kriptiven Wirklichkeitserzählungen liegt in der Schilderung von Ereignissen, die sich tatsächlich zugetragen haben, woraus sich ein Geltungsanspruch gemäß den beiden Polen ,wahr' versus ,falsch ‘ ableitet, wie dies in der Historiografie oder im Journalismus im Zuge der Rekonstruktion von Ereignissen der Fall ist. (2) Die Funktion normativer Wirklichkeitserzählungen bezieht sich auf die Beschreibung einer erwünschten Wirklichkeit. Damit wird das Ziel verfolgt, ,eine bestimmte (gesellschaftliche oder individuelle) Praxis zu regulieren, das geschieht durch exemplifikatorische Darstellungen (menschlicher Handlungen). Der Geltungsanspruch orientiert sich an der Dichotomie ,richtig handeln vs. falsch handeln““(Klein/Martínez 2009, 6). Als Beispiele für normative Wirklichkeitserzählungen führen Klein und Martínez Verhaltensratgeber, moralische Handlungsnormen sowie juristische Gesetze an. (3) Die Funktion voraussagender Wirklichkeitserzählungen liegt schließlich in der Schilderung einer erwarteten künftigen Wirklichkeit, für die allgemeine Strukturmerkmale bereits festgelegt worden sind, wie bei medizinischen Prognosen oder naturwissenschaftlich fundierten Vorhersagen zum Klimawandel. Der hier erhobene Geltungsanspruch richtet sich dabei an den beiden Polen ,plausibel` versus ,nicht plausibel' aus.

Wie zu zeigen sein wird, stellen auch die erzählten Inhalte der Moralischen Wochenschriften durch ihren ausgeprägten Bezug zur menschlichen Lebenswelt einen deskriptiven Geltungsanspruch, der aufgrund der sich erst ausbildenden bürgerlichen Gesellschaftsschicht für die Leser*innen zusätzlich normative und voraussagende Geltungsansprüche einnimmt.

\title{
2.3 Mediale Wissens- Und Welterzeugung
}

Kulturelles Wissen wird über die (Massen-)Medien generiert und disseminiert. Die in ihnen verhandelten Diskurse lenken unsere Wahrnehmung und unser Bewusstsein und nehmen somit Einfluss auf das gesellschaftliche Zusammenleben. Das Leistungsvermögen von literarischen Texten als Medien des kollektiven Gedächtnisses von Kulturen hat Astrid Erll (2005) in die Bereiche der Speicherung, der Zirkulation und des Abrufs von Wissen gegliedert. In Anlehnung an Maurice Halbwachs, der Erinnerungen stets in Abhängigkeit der ,cadres sociaux ' (sozialen Rahmungen) begreift (cf. Halbwachs 1925), spricht Erll von den ,cadres médiaux‘ (medialen Rahmungen), die auf die Erinnerung einer Person oder einer Gruppe einwirken. Erst durch mediale Repräsentationen können wir auf das spezifische Wissen von Gruppen und deren Vorstellungswelt zugreifen. Die ,medialen Rahmen des Erinnerns' ermöglichen uns, eigene und fremde Erfahrungen ins Bewusstsein zu rufen und zu deuten. Sie beeinflussen und präformieren unsere Wahrnehmung und steuern unseren Erinnerungsabruf. Ähnlich wie im vorherigen Abschnitt über das Erzählen hervorgehoben wurde, kann Literatur - die schließlich aus Erzählungen besteht - als Speichermedium einer Erinnerungskultur normativ und formativ rezipiert werden und somit eine kulturelle Dimension annehmen. Als Zirkulationsmedium konstruiert und vermittelt Literatur Inhalte des kollektiven Gedächtnisses (wie Geschichtsbilder, Werte, Normen, Identitäten), wobei wir diesen Inhalten eine gewisse Realitätsnähe zugestehen müssen, um sie als glaubhaft und normativ aufzufassen. Als Abrufmedium funktioniert Literatur schließlich dann, wenn wir allein durch die Nennung von bestimmten Texten an bestimmte Geschichtsbilder, Werte oder Normen denken (cf. Erll 2005, 254-263). 
Im Sammelband Cultural Ways of Worldmaking (2010) greifen Ansgar Nünning, Vera Nünning und Birgit Neumann den konstruktivistischen Ansatz nach Goodman auf und machen ihn für die Kultur- und Literaturwissenschaften fruchtbar, indem sie aufzeigen, wie Welten und Wissen mithilfe von Erzählungen und Medien erzeugt, strukturiert und verändert werden. Erzählungen und Medien verstehen sie als kognitive Werkzeuge, die uns helfen, uns und unsere Lebenswelt zu verstehen. Sie sind als spezifische Ausdrucksformen einer Kultur zu begreifen, die eine Kultur nicht nur hervorbringt, sondern die umgekehrt diese auch gleichzeitig formen (Nünning/Nünning 2010, 15-16). Im Verbund mit Erzählungen produzieren und verbreiten Medien folglich kulturelles Wissen, Werte und Normen. Sie zirkulieren Sinnangebote, Wahrheiten und Meinungen, die sich auf uns, unsere Mitmenschen und unseren Umgang miteinander auswirken. Dabei zeigt sich, dass unterschiedliche Medien und Gattungen jeweils eigene Ausprägungen von Erzählungen hervorbringen und ihre Welten inhaltlich, strukturell und sprachlich jeweils anders konstruieren und somit auch unterschiedliche Ausdrucks- und Sinnstiftungsmöglichkeiten anbieten. Spricht man von ,Gattungen“, so muss man sich vor Augen halten, dass es sich dabei um ,pragmatische Konstrukte handelt, die von Wissenschaftlern nach Maßgabe bestimmter Differenzierungskriterien konstruiert werden“ (Nünning/Rupp 2012, 9). Da Gattungen historisch gewachsen sind, sind sie im Laufe der Zeit auch immer wieder Veränderungen unterworfen, das heißt, sie sind „keine überzeitlichen, sondern geschichtlich bedingte Verständigungsund Konventionsformen“ (ibid., 17). ${ }^{20}$ So veränderte sich die Medienlandschaft im Laufe der Jahrhunderte vom Menschmedium zum Druckmedium und im 21. Jahrhundert zu den sozialen Medien. Mit jedem gesellschaftlichen Wandel entwickelten sich Medien vor allem als „Antworten auf aktuelle soziale Bedürfnisse“ (Bösch 2011, 227) weiter. Zum Beispiel werden heute die Entwicklung des Buchdrucks sowie die Verbreitung des Humanismus und die Reformationsbewegung Martin Luthers (14831546) als interdependent betrachtet, ohne deren Zusammenwirken die konfessionelle Spaltung wohl nicht bereits im 16. Jahrhundert stattgefunden hätte.

Auch der Sozialwissenschaftler Benedict Anderson schreibt dem Buchdruck eine besondere Rolle in der medialisierten Herstellung von Gemeinschaften zu. In seinen Überlegungen zur Erfindung der Nation (2005), einer ins Deutsche übersetzten Monografie, deren englisches Original unter dem Titel Imagined Communities 1983 erschien, beobachtet er, dass auch postkoloniale Gesellschaften ,sich zunächst national definierten“ und dass „,sie Instrumente einsetzten und Wirklichkeitsvorstellungen okkupierten, die sie glaubwürdig als nationale Gesellschaften darstellten“ (Mergel 2005, 283). Aus dieser Beobachtung heraus entwickelt Anderson den Gedanken, dass es sich bei jeder Nation um eine vorgestellte, territorial begrenzte, souveräne Gemeinschaft, eine sogenannte ,imagined community ‘ handle, deren Mitglieder sich zusammengehörig fühlen, ohne sich alle persönlich zu kennen. Als unhinterfragte, gegebene kulturelle Bezugssysteme gaben religiöse Gemeinschaften und dynastische Reiche bis ins 18. Jahrhundert hinein den Menschen in ihrem Bestreben nach Konsistenz einen

20 So reflektieren bereits in der Antike Platon (ca. 427-348/347 v. Chr.) und Aristoteles (384322 v. Chr.) über ihre Gattungsvorstellungen und die variierenden Effekte von Gattungen (cf. Zymner 2010, 199-200), die in den Folgejahrhunderten immer wieder aufgegriffen und abgewandelt werden. 
überzeitlichen Rahmen. Die „Idee der Nation“ (Anderson 2005, 20) konnte schließlich in jenen Kulturräumen entstehen, in denen sich das Weltbild in dreierlei Hinsicht zu ändern begann: (1) Man kehrte sich von der Vorstellung ab, es gäbe eine besondere Schriftsprache, mit der man einen privilegierten Zugang zu einer ontologischen Wahrheit hätte. (2) Der Glaube an eine göttlich entsandte Herrscherfigur wurde immer mehr in Frage gestellt und ging schließlich verlustig. (3) Durch den vermehrten Druck von Romanen und Tageszeitungen änderte sich die Zeitvorstellung der Menschen von einer homogenen, leeren Zeit (Walter Benjamin) in eine parallele Zeitvorstellung, in der unterschiedliche Ereignisse parallel stattfinden konnten. Diesen Veränderungen ging die Etablierung einheitlicher Schriftsysteme voraus, die den Sprachenpluralismus in den verschiedenen Dynastien beendete und eine Schriftsprache durchsetzte, die zum Fundament für ein Nationalbewusstsein werden sollte. Verbreitet wurde die jeweilige Einheitssprache als Kommunikationsgrundlage für Sprechergruppen derselben Sprachen durch das kapitalistische Unternehmertum des Buchdrucks. Gleichzeitig wurde man sich gewahr, dass man zu einer Gruppe gehörte, die dieselbe Sprache teilte, und dass es andere Menschen gab, die einer anderen Sprachgruppe angehörten. Ferner entstanden neue Machtsprachen, das heißt, gewisse Sprachen wurden aufgewertet und andere abgewertet. Für Anderson ist die Zeitung das geeignete technische Mittel, um ein kollektives Bewusstsein von Nation entstehen zu lassen. Durch die geteilte mediale Praxis des morgendlichen Zeitunglesens entsteht eine Gemeinschaft beziehungsweise ein Gemeinschaftsgefühl. Dieses resultiert aus dem erwachsenden Bewusstsein unter den Leser*innen, dass Abertausende weiterer Personen gerade dasselbe tun und dass die unterschiedlichsten Ereignisse, über die berichtet wird, gleichzeitig stattfinden. In der erweiterten Auflage aus dem Jahr 1996 geht Anderson schließlich auch auf die wesentliche Bedeutung von Erzählungen in der Konstruktion von Nationen ein. Wie die Erzählung des eigenen Lebens ist die Erzählung einer Nation ,,in eine homogene, leere Zeit hineingestellt“ (Anderson 2005, 206), die es mit Ereignissen zu befüllen gilt, um ein gemeinschaftsstiftendes „Narrativ der ,Identität““ (ibid., 207) zu erzeugen.

In ähnlicher Weise verhält es sich mit der Ausbildung anderer, nicht real erlebter Gemeinschaften. So zum Beispiel stützt sich die Ausbildung eines bürgerlichen Gemeinschafts- und Differenzgefühls unter anderem auf die soziale Praxis der Lektüre der Moralischen Wochenschriften, in denen „das Publikum, das derlei liest und bespricht, [...] sich selbst zum Thema [hat]“ (Habermas 1990, 107). Wie aus dem Folgenden ersichtlich wird, speisen sich die spectatorialen Erzählungen aus der Lebenswelt der Leser*innen und zeichnen gleichzeitig ein normatives Idealbild der bürgerlichen Welt und ihrer Subjekte. Vor dem Hintergrund des historischen Geschlechterwissens wird in den Periodika ein stereotypes Rollenarsenal weiblicher und männlicher Figuren präsentiert, das als Grundlage für die Ausbildung einer idealtypischen Bürgerlichkeit fungiert. 